\title{
Some Properties of Coherent Smith-Purcell radiation from electron bunches and gratings of finite dimensions.
}

\author{
I.V. Konoplev ${ }^{\mathrm{a}}$, F. Bakkali Taheri ${ }^{\mathrm{a}}$, G. Doucas ${ }^{\mathrm{a}}$, A. Reichold ${ }^{\mathrm{a}}$, R. Bartolini ${ }^{\mathrm{a}, \mathrm{c}},{ }^{2}$ N. Delerue ${ }^{\mathrm{b}}$, C. Clarke $^{\mathrm{d}}$, \\ V. Bharadwaj ${ }^{\mathrm{d}}$. \\ ${ }^{a}$ JAI, University of Oxford, OX1 3RH, UK \\ ${ }^{\mathrm{b}}$ LAL, University Paris-Sud XI, 91898 Orsay, France \\ ${ }^{c}$ Diamond Light Source Ltd., Didcot, OX11 0QX, United Kingdom \\ ${ }^{\mathrm{d}}$ SLAC National Accelerator Laboratory, Menlo Park, CA 94025, USA
}

\begin{abstract}
There is a strong interest in the development of single shot diagnostics of fs relativistic electron bunches within research areas including $\mathrm{THz}$ sources of radiation. The goal of the work presented is to understand the properties of coherent SmithPurcell radiation to enable the reconstruction of the longitudinal profile of a fs-long electron bunch.
\end{abstract}

\section{INTRODUCTION AND BACKGROUND}

There is currently significant interest in the development of compact particle accelerators capable of producing fs relativistic electron bunches to act as drivers for $\mathrm{THz}$ sources of coherent broad band (ps-long) radiation. To support the progress in these areas a non-invasive, femtosecond (fs) electron beam diagnostic is required. There is, at present, no such device available [1-3]. The goal of this project is to study coherent Smith-Purcell radiation (cSPr) through the use of PiC codes. This will enable us to build a detector capable of profiling fs-long electron bunches in a single shot, thus offering non-invasive and real time monitoring, scalable to a wide range of conditions.

\section{RESULTS}

Coherent Smith-Purcell radiation is emitted by a corrugated metallic surface (grating) over a wide range of angles, when a relativistic electron beam passes in the vicinity of the grating (fig.1a). The coherence of the radiation is achieved by selecting the grating period $d$ to be larger than the longitudinal size of the beam [4]. In this case electron beam emits cSPr (signal) over a broad spectral range. The incoherent radiation from a relativistic beam is also emitted but its intensity is only proportional to the number of electron per bunch while in the case of coherent radiation the intensity of the radiation is proportional to square of the electrons in the bunch. Another advantage of this method is the correlation between angular and spectral distributions of the emitted radiation which is given (in the far field) by the following relationship:

$$
\lambda \approx d(1 / \beta-\cos \theta) / m \text { (1) }
$$

where $l$ is the grating period, $m$ is the diffraction order, and $\theta$ is the emission angle ( $\mathrm{x}-\mathrm{Z}$ plane, fig.1a) with respect to the electron beam propagation direction. Locating the detectors at specific angles and using condition (1) the spectrogram of the pulse (its FFT) can be measured and using appropriate mathematical techniques, one can reconstruct the temporal structure of the bunch form this spectral-angular distribution. In this presentation we will discuss such properties of cSPr as polarization, its spectral characteristics and radiation patterns.
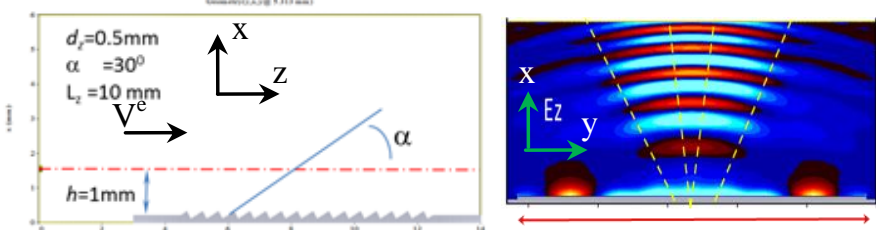

Figure 1: (a) Full 3D Numerical model of a broadband cSP radiator observed using $\mathrm{PiC}$ code $\mathrm{MAGIC}$; (b) Contour plot of $E_{z}$ field component generated by the fs-bunch (x-y plane) at the middle of the grating.

Fig.1a shows a 3D model of an 1D aluminium grating of period $0.5 \mathrm{~mm}$, length and width of $10 \mathrm{~mm}$ and 30 degree blaze angle. The bunch parameters were assumed to be similar to those available at FACET SLAC. An example of the radiation pattern (in $\mathrm{x}-\mathrm{y}$ plane) observed is shown in fig. $1 \mathrm{~b}$. It was expected that the cSPr is strongly collimated in $\mathrm{x}-\mathrm{y}$ plane with the aperture and divergence angle $\varphi$ (in $\mathrm{x}-\mathrm{y}$ plane, dotted lines) for a finite size beam defined by beam's "footprint" [5] and relativistic Lorentz factor $\gamma(\gamma=40000$ at FACET and $\varphi<1$ degree) [6]. However, as seen in the figure, the angle is much larger due to excitation of higher order modes along the corrugation [5]. We also note that this will affect the degree of polarization measured by a detector with a finite acceptance angle, as well as the overall signal yield.

\section{REFERENCES}

[1] I. Wilke, et al.,"Single-Shot Electron-Beam Bunch Length Measurements", Phys. Rev. Lett. 88, 124801, 2002.

[2] M. Veronese, et al.," Absolute Bunch Length Measurement Using Coherent Diffraction Radiation", Phys. Rev. Lett. 110, 074802, 2013

[3] Y. Glinec, et al., Observation of Fine Structures in Laser-Driven Electron Beams Using Coherent Transition Radiation”, Phys. Rev. Lett. 98, 194801, 2007.

[4] R. Bartolini, et al., "Electron bunch profile reconstruction in the few fs regime using coherent Smith-Purcell radiation", Journal of Instrumentation, 7, P01009, 2012.

[5] I.V. Konoplev et al., "Relativistic Electron Beam Excitation of Surface Fields in Artificial Materials Based on One- and Two-Dimensional Periodic Structures", IEEE Trans. on Plasma Sci., 39, p.2610, 2011.

[6] G. Kube, "Calculation of SP radiation from a volume strip grating", Nucl. Instrum. and Methods in Phys. Res., B 227, p.180, (2005) 